\title{
Error médico y eventos adversos
}

\author{
PATRICIA MENA N. ${ }^{1}$ \\ 1. Profesora Adjunta Asociada, Módulo Docente Pontificia Universidad Católica de Chile, Servicio de Neonatología, \\ CA Dr. Sótero del Río.
}

\begin{abstract}
Medical Errors and Adverse Events: New Paradigms?

The literature is reviewed about the perception and attitude of physicians and patients in relation to medical errors, specially in the context of public health service. The actual tendency is to consider them as part of the medical process with a systemic approach, where monitoring and prompt recognition is important in enhancing the quality and security of the hospitalized patients. In ethics, the new paradigm is to inform medical errors. Eventhough legally it is not clear, there have been international advances in this topic. Some recommendations are described to confront these situations with patients and understand their reactions. In the future, more transparency is expected.
\end{abstract}

(Key words: Medical error, adverse events, ethics).

Rev Chil Pediatr 2008; 79 (3): 319-326

\section{RESUMEN}

Objetivo: Revisar la literatura en relación a la actitud y la percepción del equipo de salud y los pacientes en relación a error médico y eventos adversos en sistemas de salud, especialmente en el contexto hospitalario de los sistemas públicos de salud. La tendencia actual descrita en la literatura es considerar los errores como parte del proceso médico, con un enfoque sistémico, cuya vigilancia y reconocimiento es fundamental en la mejoría de la calidad y la seguridad del paciente hospitalizado. Desde la ética, el nuevo paradigma de informar errores está muy establecido pero el marco legal no es tan claro, aunque internacionalmente hay cambios al respecto. Se describen algunas recomendaciones para asumir la situación frente a los pacientes y entender sus reacciones. Se espera una mayor transparencia en la información a futuro.

(Palabras clave: Errores, eventos adversos, ética, pacientes, médicos, sistemas, demandas).

Rev Chil Pediatr 2008; 79 (3): 319-326

Trabajo recibido el 28 de febrero, devuelto para corregir el 08 de abril de 2008, segunda versión el 18 de abril de 2008 , aceptado para publicación el 05 de mayo de 2008.

Correspondencia a:

Patricia Mena N.

E-mail: pmenanani@gmail.com 


\section{Introducción}

Los errores médicos son parte inseparable del ejercicio de la Medicina. ¿Cómo se enfrenta hoy esta realidad?.

¿Cuáles son las recomendaciones actuales para prevenir los errores?

¿Qué pasa en los hospitales donde los errores pueden ser producidos por una sucesión de situaciones provocando ocasionalmente graves consecuencias para el paciente?

¿Pueden los pacientes entender un error que podría haber influido o ser determinante en una evolución desfavorable?

La mayoría de nosotros nos enfrentamos personalmente más de alguna vez a los errores médicos tanto desde el lado de ser profesional, como desde el ser pacientes. Cometer errores es inherente a la condición humana, especialmente en ámbitos en que la incertidumbre de ciertas variables en la toma de decisiones y la variabilidad misma de la biología humana, impide un resultado exacto. Nos damos cuenta de los errores retrospectivamente, pero al momento de cometerlos, no somos concientes de estarlo produciendo. La apreciación del error es histórica, podemos prevenir o disminuir su frecuencia, pero nunca dejaremos de cometerlos.

Objetivo: Revisar la literatura internacional en Pubmed, y la nacional en Scielo y Google en relación a la actitud y la percepción del equipo de salud y los pacientes en relación a error médico y eventos adversos en sistemas de salud.

\section{El Nuevo paradigma}

Si bien la literatura médica desde hace muchos años presenta algunos estudios y comentarios sobre el tema, en especial sobre los errores de medicación, la publicación del Instituto Americano de Medicina en 1999 del reporte "To Err is Human" es la que provoca un creciente número publicaciones, incluyendo el análisis de la prensa no especializada, con un eco importante en los medios. En Chile, se realizó un panel en la Reunión de la Región Chilena de American College of Physicians con publicación en la Revista Médica de Chile el año 2001 2-6. Algunos congresos sobre gestión de calidad han analizado el tema y hay publicaciones de manejo de errores en cirugía $^{7,8}$. La información nacional se concentra en centros privados y hospitales clínicos como conferencias o talleres mucho más que en los servicios públicos hospitalarios ${ }^{9-11}$. Entre los cirujanos está mucho más incorporada la noción de error y su reconocimiento, y en los formularios de consentimiento informado para cirugía se tiende a detallar muchas consecuencias inesperadas, lo que se observa menos en las especialidades médicas ${ }^{2-8}$.

\section{¿Qué se entiende por error médico?}

"Error: Concepto equivocado, juicio falso, acción desacertada. Diferencia entre un valor medido o calculado y el valor real, equivocación de buena fe." 12 .

Hay una gran cantidad de términos utilizados como sinónimos, que en parte evitan la carga de responsabilidad o culpabilidad que frecuentemente se asocia al término error médico: reacciones adversas, complicaciones del cuidado, eventos adversos, eventos adversos prevenibles, eventos adversos potenciales, eventos centinela, eventos serios reportables, consecuencias no intencionales, resultados no terapéuticos, evento desfavorable.

La definición más frecuentemente citada es la del Institute of Medicine: "Falla de una acción planeada para ser completada según la intención (error de ejecución) o el uso de un plan equivocado para alcanzar un objetivo (error de planeamiento)"'. La definición de: "decisión diagnóstica o terapéutica o procedimiento que, dado el momento y las circunstancias de la ocurrencia puede ser considerado erróneo por pares calificados y con experiencia" parece más clara. Esta definición excluye las consecuencias y el curso natural de la enfermedad y excluye decisiones hechas bajo circunstancias extremas como puede ser una atención de urgencia con gran presión clínica ${ }^{13}$.

Más recientemente se ha distinguido el error médico del evento adverso, considerando este último como la injuria producida por el error médico ${ }^{14}$. Además se ha establecido la distinción del "Casi error" que se caracteriza por la potencial (pero no real) injuria al paciente, pero que es muy importante de reconocer, analizar y 
prevenir para que en el futuro no se presente como un error ${ }^{13,14}$

A nivel hospitalario se ha descrito como evento centinela al "hecho inesperado, no relacionado con la historia natural de la enfermedad, que produce la muerte del paciente, una lesión física o psicológica grave o el riesgo de sufrirlas a futuro"15. Estos eventos centinela se han incorporados en los procesos de evaluación de calidad de los hospitales. Así se realizan auditorias de la muerte materna, las reacciones transfusionales, la transfusión de grupo sanguíneo equivocado, las infecciones intrahospitalarias y las reacciones adversas graves a medicamentos. Representan una mínima cantidad de los eventos adversos potenciales significativos en una hospitalización, pero su reporte y análisis permite prevenir parte importante de los eventos adversos ${ }^{12,15}$.

Desde el punto de vista ético, el enfrentamiento de los errores se enmarca en el "principio de beneficencia", que conduce a promover el beneficio del paciente como propósito fundamental, llevando implícito el principio de "no maleficencia", es decir, evitar cualquier acción que pudiera causarle daño. Pero esto no es suficiente $^{12}$. El imperativo ético de revelar los errores, incluso los casi errores o aquellos sin consecuencias significativos ha sido establecido por diversas instituciones a nivel internacional como la American Medical Association Council on Ethical and Judicial Affaire, Institute of Healthcare Improvement, National Academy for State Health Policy y otras ${ }^{16-20}$. A nivel nacional se establece esta recomendación en la Carta Médica de Chile de la Sociedad Médica de Santiago ${ }^{21}$. En la declaración de derechos y deberes de los pacientes, no se contempla originalmente que el paciente tenga el derecho a ser informado de los errores producidos en el transcurso del tratamiento ${ }^{22}$. Si bien los actuales estándares de acreditación del Ministerio de Salud se refieren al registro y evaluación de los eventos adversos, no se menciona nada sobre la comunicación de éstos al paciente o su familia ${ }^{23}$.

\section{¿Cómo reaccionan los médicos en estas nuevas normativas?}

La actitud del médico frente al error médico se ha ido modificando, pero aún hay una gran resistencia a reconocer errores. La experiencia compartida de cometer errores crea un poderoso sentimiento de mutua empatía que a menudo lleva a entender, perdonar y a una fuerte tendencia de falta de crítica, descrita como una "conspiración de tolerancia" ${ }^{24}$. Los médicos se justifican con argumentos sobre la incerteza en medicina, las limitaciones humanas, las características de las enfermedades y de los pacientes, los problemas de la organización, fatiga y problemas personales.

Mizrahi, cita tres mecanismos para defender el error médico: la negación, el descontar y el distanciamiento. Con la negación se define la práctica de la Medicina como un arte de áreas grises, en que los errores no son errores. El descontar considera la atribución de responsabilidades a factores externos o circunstancias fuera de control como la enfermedad o los pacientes mismos, esto se da especialmente en los casos en que el paciente pertenece a algún grupo tradicionalmente estereotipado negativamente, como los pacientes con problemas de abuso de drogas o los portadores de enfermedades como SIDA. Cuando los errores no pueden ser negados ni descontados se usan técnicas de distanciamiento como "todos cometemos errores", "no era posible de prever" 24

Los médicos concientes y clínicamente competentes cometen errores que reconocen, lamentan, con los cuales aprenden y conviven ${ }^{24}$. Ser explícito con el paciente sobre los errores representa el cambio de paradigma para los médicos. A pesar de que la mayoría de los médicos reconoce un hipotético error, una minoría de ellos está dispuesto a entregar detalles a su paciente acerca de éste; sobre todo si es un error poco aparente y de un efecto trivial ${ }^{25}$. El temor de las demandas, la amenaza a su prestigio, sentirse avergonzado o no saber cómo efectivamente informar a los pacientes puede influir en el silencio de los médicos sobre sus errores $^{26,27}$.

Un estudio con médicos de atención primaria españoles, sobre los errores cometidos el último año, describe a éstos como: negadores, aquellos que consideran no han cometido error, un $28 \%$ en esta muestra; perceptivos los que 
reconocen al menos un error, el 67\%; y los hiperperceptivos que reconocen 28 o más errores el último año, que fueron un $7 \%$. Respecto a la comunicación al equipo, los médicos jóvenes, en contraste con los mayores, comunican más frecuentemente sus errores. Los hiperperceptivos tienden a tener mayores reacciones emocionales después de cometer errores ${ }^{28}$.

En este grupo de médicos los errores reportados más frecuentes fueron: la prescripción de un medicamento inadecuado para la enfermedad de base, el retraso diagnóstico en un escenario clínico de paciente neoplásico y la interpretación de manera errónea de una prueba complementaria. Los errores fueron atribuidos por los médicos a la presión asistencial, la mala organización de la asistencia y el poco apoyo a la formación continua ${ }^{28}$.

Existe poca información sobre la percepción y actitudes de los pediatras frente a los errores. Un cuestionario realizado en un estado norteamericano muestra que solo un $7 \%$ declara no haber estado envuelto en ningún error, un $39 \%$ en errores serios (daño permanente o transitorio con potencial compromiso vital) y un $72 \%$ en errores menores (que no causa daño permanente ni es potencialmente mortal) $)^{14}$. La mayoría de estos pediatras aprueba el reporte de los errores, pero sólo la mitad conoce el sistema para ello en sus instituciones y el $9 \%$ afirma la inexistencia de éste. Un $92 \%$ afirma haber usado un mecanismo formal para reportar un error . El informe del error se realiza más cuando el médico piensa que este es un aspecto importante del cuidado en salud y cuando piensa que la responsabilidad es más del sistema que individual. Los factores que favorecen el reporte de errores por parte de los pediatras son: el que la información sea utilizada para mejorar el sistema, que se mantiene la confidencialidad, un sistema de reporte no punitivo, que el proceso no tome más de 2 minutos y que se realice en el mismo lugar ${ }^{14}$.

Indudablemente la cultura organizacional es muy variable de un centro a otro y es probable que una jefatura negadora no considere los errores, y cuando se le muestran siempre encuentra explicaciones para ellos fuera del servicio: "la literatura no dice nada al respecto", "las normativas no se refieren a ello".

\section{¿Cuál es la mirada de los pacientes?}

Ante una situación adversa es habitual que una persona busque o atribuya responsabilidades, necesita encontrar un culpable; es un sentimiento atávico para poder tener control sobre su propia vida.

En la cultura occidental, de origen judeo cristiana, las tradiciones de confesión, arrepentimiento y perdón están profundamente arraigados, aún en aquellas culturas más seculares. No es raro entonces que los pacientes y la familia frente a los eventos adversos esperan una explicación, una disculpa y el que se les asegure que un incidente similar no se repetirá ${ }^{29}$.

Hay una creciente percepción de que el error médico es común y que es fundamentalmente tolerado por la profesión médica, cuando no protegido $^{24}$.

Los pacientes consideran errores médicos la evolución no anticipada, las dificultades de comunicación y violaciones de la confianza. La percepción de cómo el paciente es tratado por la comunidad médica después de una evolución inesperada afecta la percepción misma del evento. Si el equipo médico muestra acciones para corregir el error, es más fácil que el paciente perdone el error médico. Cuando la preocupación del usuario no es direccionada o validada, es más probable que se agrave la percepción de un error. Adoptar un ambiente en que los pacientes puedan conversar abiertamente con el equipo médico acerca de los errores y dando a los clínicos la oportunidad de de revisar cada caso y ofrecer educación sobre el curso natural de la enfermedad, complicaciones y eventos adversos no prevenibles, puede modificar la percepción de los pacientes sobre los errores ${ }^{30}$.

Los estudios respecto a las reacciones en situación de crisis muestran que la reacción a las crisis provocadas por la naturaleza es diferente de aquellas derivadas o atribuidas por la persona a causas humanas. Estas últimas provocan mucha rabia, generan una sensación de injusticia, dañan la confianza de las personas y siempre existe la percepción de que podrían haberse evitado ${ }^{30,31}$.

Los pacientes y sus familias son capaces de identificar errores e injurias durante la hospitali- 
zación. Esta es una información útil para el cuidado clínico y para desarrollar iniciativas que mejoren la seguridad de los pacientes. También son capaces de identificar las condiciones de riesgo, como la presencia de personal insuficiente. Comprometer a los pacientes en el esfuerzo de identificar y prevenir errores ofrece una promisoria estrategia en el avance por la seguridad del paciente ${ }^{32}$.

\section{¿Cómo congeniar una práctica ética con un riesgo legal?}

"Hacer lo que no se sabe que no se debe hacer" es ignorancia.

"No hacer lo que se sabe que se debe hacer" es negligencia.

"Hacer lo que se sabe que no se debe hacer" es dolo ${ }^{12}$.

Desde el punto de vista legal la situación es compleja. En especial en momentos de cambio de la estructura judicial. También en lo administrativo. El sistema público tiene una larga tradición sumarial de identificar culpables individuales en errores sistémicos, por lo cual es complejo plantear revelar ampliamente los sucesos tras un evento adverso. Es un camino lento, que debe iniciarse con la prevención sistemática, que requiere, previamente, la identificación de cada evento.

El entorno legal, judicial y comunicacional en Chile no fomenta una discusión abierta sobre los eventos adversos. Por el contrario, se mantiene la tendencia de ocultar su ocurrencia, subnotificar los casos a las instancias superiores del propio establecimiento, debido al temor que genera la posibilidad de verse expuesto a un daño irreparable a la imagen del profesional o a una acción judicial ${ }^{33}$.

En Estados Unidos, el año 2002 un 36\% de las instituciones de salud tenían establecida una política de informe de errores, esta cifra ha aumentado a $69 \%$ el año 2005. Esta información se ha constituido en el componente central de la calidad en el cuidado médico ${ }^{26}$. Aproximadamente dos tercios de los estados en Estados Unidos tiene leyes que protegen la expresión de disculpa no permitiendo que esta sea utilizada en una demanda, pero no protegen la información de causa o de falta.

Por otra parte, aunque esta información pue- da o no ser utilizada legalmente permite que los abogados dedicados a demandar puedan encontrar elementos para establecer demandas ${ }^{26}$.

Estudios en medicina y otras áreas sugieren que los individuos prefieren disculpas explícitas, y que, en general, estas disculpas disminuyen el riesgo de juicios y promueven menores y más rápidos acuerdos cuando los juicios se realizan. Lo importante ha sido el reconocimiento, el hacer explicito la inevitabilidad de la existencia del error médico y la necesidad de asumirlo institucionalmente para avanzar en la prevención y en la generación de un mayor nivel de seguridad para el paciente. Una comunicación abierta sobre los errores es una de las bases del movimiento por la seguridad del paciente. Develar los errores con daño es recomendado como una obligación ética y como exigencia de los nuevos estándares de acreditación a nivel internacional ${ }^{15,34}$.

Algunos hospitales americanos mantienen, desde 1987, una política de continua relación entre el hospital y el paciente, con disculpas explicitas al paciente dañado ya sea por accidente o negligencia y una justa compensación por el daño producido. Con esto han logrado reducir moderadamente los gastos por juicios de pacientes, ya que los costos de los juicios se han miminizados y se disminuye la rabia y deseo de venganza que muchas veces lleva a los pacientes a una demanda ${ }^{35}$.

\section{¿Como reducir errores médicos a nivel institucional?}

Lo más importante del nuevo paradigma es el enfoque institucional de los errores médicos.

El primer gran factor fundamental a nivel institucional es que se cree una cultura profesional de considerar los errores como existentes, pero que pueden ser prevenidos a futuro con un reconocimiento, un análisis y la modificación de las prácticas clínicas identificadas como responsables. La creación de un ambiente protegido, libre de culpas, que promueva el reporte sistemático de los eventos adversos serios permitirá tener una valiosa información para el trabajo preventivo ${ }^{13}$. El uso de reportes anónimos de incidentes en los servicios hospitalarios ha permitido identificar, analizar y crear políticas de prevención. Además permite cuan- 
tificar los hechos y establecer metas en el tiempo. El equipo de salud debe ir entendiendo la importancia de informar sin ser penalizado por ello ${ }^{36}$.

En el análisis de eventos adversos generalmente se observan múltiples momentos en los que el evento pudo ser evitado. La mayoría de los errores tienen que ver con fallas institucionales más que en errores personales ${ }^{35,36}$.

Se debe promover un cambio cultural, a través de desarrollar un pensamiento disciplinado, que conduzca a la investigación y análisis sistemático de las causas de los eventos adversos y al trabajo organizado para su prevención $^{12}$. Ello implica actuar sobre individuos, equipos, tareas, lugares, normas e instituciones, para reducir la incidencia de estos problemas y disminuir sus riesgos o, incluso, hacer más aparentes los errores imprevisibles para que sean advertidos con sentido profiláctico ${ }^{2}$.

Toda institución hospitalaria o de atención abierta debe contar con un comité de manejo de riesgo. Este debe identificar los hechos en un accidente, posible negligencia o mal práctica. La literatura norteamericana describe que la investigación incluye la entrevista con los médicos, y el personal. Posterior al análisis se llama al paciente o a la familia y se le solicita concurrir porque ha habido un problema y puede concurrir con su abogado si lo desea. Se realiza reunión con el Director médico, el abogado de la institución y el profesional a cargo del comité de calidad donde se informa detalladamente de lo ocurrido, incluyendo las personas involucradas que han sido previamente informadas. El énfasis está en las excusas de la institución y su preocupación por el evento y en las medidas que se han tomado para evitar eventos similares. El comité ofrece tratamiento, si es necesario, y compensación económica cuando corresponde ${ }^{36}$. Un adecuado sistema de seguros profesionales e institucionales debería contemplarse para este efecto.

Aunque el evento adverso hubiera ocurrido por causas diferentes a un error médico, como consecuencia de la variabilidad biológica de los humanos, fallas en los equipos, en el mantenimiento, en la organización, en la comunicación con el paciente y familiares, accidentes, en la coordinación, en la capacitación del personal, etc., el equipo médico es el responsable de prevenirlos, evitarlos, identificarlos con oportunidad y limitar el daño que pudieran producir al paciente, si queremos garantizar una atención médica con calidad, segura y con respeto del principio ético de beneficencia ${ }^{12}$.

En los servicios públicos de salud en los que ocurren muchos eventos adversos de múltiples causas, la ausencia de una política institucional que de respuesta a estas inquietudes en el marco cultural descrito, muchas veces con puertas cerradas frente a la necesidad de explicaciones coherentes y de demostrar preocupación por el hecho, favorecerá la judicialización de la Medicina. La incorporación de las oficinas de información, reclamo, sugerencia y felicitaciones del Ministerio de Salud y de los servicios y hospitales públicos (OIRS) quiere mejorar los problemas de comunicación pero no incluye una comunicación directa de los médicos encargados con el paciente y su familia frente a un error médico, ni ha habido un entrenamiento necesario para ello ${ }^{37}$.

Revelar un error es similar a dar malas noticias. Debe incluir una sincera descripción de la naturaleza del error, sus consecuencias, y las acciones correctivas emprendidas. La expresión de remordimiento y una disculpa son importantes. Debe darse amplia posibilidad para preguntas. El médico debe entender que el paciente va a enojarse y no ponerse a la defensiva. Se debe promover una atmósfera de discusión abierta y el reconocimiento de su inevitabilidad $^{38}$.

Para disminuir errores médicos se debe promover el uso y disponibilidad de los análisis de medicina basada en evidencias, las guías clínicas, la epidemiologia; mantener una buena competencia profesional a través de programas de formación continua, el trabajo en equipo, un buen manejo de la ficha clínica, la estabilidad del equipo de salud y una óptima supervisión del personal en formación ${ }^{12}$.

\section{Conclusiones}

El nuevo paradigma es un gran desafío. Paralelamente a éste debe realizarse una gran intervención educativa que acerque a la pobla- 
ción a la realidad de la medicina, su falibilidad, un adecuado uso de programas de difusión médica, mostrando éxitos y fracasos, evitando dar la impresión de omnipotencia del desarrollo médico y por otro lado un manejo noticioso más ponderado de los casos de eventual error médico. La existencia de mediadores en salud de la nueva justicia procesal podría mejorar la comunicación entre pacientes y equipo médico cuando ésta se ha quebrado.

Desconocer los errores médicos en un momento en que cualquier profesional con manejo en Internet puede adquirir rápidamente una enorme cantidad de información con la cual enfrentar al equipo médico, no es la respuesta más adecuada. Transparentar el ejercicio médico aparece como la alternativa apropiada, como se comenta en las publicaciones revisadas. Aprovechar los errores como una fuente de aprendizaje y cambio permite mantener una mejoría continua en la calidad de la atención.

Gallagher señala: "Ha comenzado una transformación sobre cómo la profesión médica comunica a los pacientes acerca de los errores. Dentro de una década, la información franca y completa de los eventos a los pacientes será la norma, más que la excepción... Esta conducta será un paso crítico para restaurar la confianza pública en la honestidad e integridad del sistema de salud ${ }^{26}$.

Gallagher es muy optimista, tal vez tome más tiempo en generalizarse esta conducta, probablemente aparezcan otras prácticas y dificultades, pero por ahora, éste parece ser el rumbo más adecuado.

\section{Referencias}

1.- Kohn L, Corrigan J, Donaldson M: To erris human. Building a safer health care system. National Academy Press ,Washington D.C. 2000 http://www.nap.edu/ openbook.php?Isbn $=0309068371$

2.- Rosselot E: Errores en medicina. Rev Méd Chile 2001; 129: $1455-8$

3.- Goic A: Fuentes de error en clínica. Rev Méd Chile 2001; 129: 1459-62.

4.- Carvallo A: Consideraciones éticas sobre el error en medicina. Rev Méd Chile 2001; 129: 1463-5.

5.- Szantho G: Definiciones respecto al error en medicina: some definitions. Rev Méd Chile 2001; 129: 14669.
6.- Sutil L: Manejo de los errores en medicina. Rev Méd Chile 2001; 129: 1470-2.

7.- Campaña $G$ : Errores médicos en el ambiente quirúrgico. Como prevenirlos. Parte I. Generalidades Rev Chil Cir 2006; 58: 235-8.

8.- Campaña G: Errores médicos en el ambiente quirúrgico. Como prevenirlos: Parte II. Errores de medicación en el pabellón quirúrgico. Rev Chil Cir 2006; 58: 305-7.

9.- OPS celebra primer taller para defensores de pacientes contra errores médicos en las Américas http://www.un. org $/$ spanish $/$ News/fullstorynews. Asp?Newsid=6881 \& criteria $1=$ Latina

10.- Vargas V: Comité de Ética. Revista Hospital Clínico Universidad de Chile 2003; 14: 162-71. http://www. redclinica.cl/publicaciones/volumen14-2/comitesetica. pdf

11.- Chomali M, Miranda F: Gestión de riesgos en la atención de salud: hacia una cultura de la calidad basada en la seguridad. Revista Clinica Las Condes 2003; 14: 4 http://www.clinicalascondes.cl/Area_Academica/ Revista Medica Octubre 2003/articulo 006.htm

12.- Aguirre-Gas H, Vázquez-Estupiñán F: El error médico. Eventos adversos. Cir Ciruj 2006; 74: 495-503.

13.- Murphy JG, Steel A, Mcevoy MT, Oshiro J: Journal reporting of medical errors. The wisdom of Solomon, the bravery of Achilles and the foolishness of Pan. Chest 2007; 131: 890-6.

14.- Garbutt J, Brownstein DR, Klein DJ, et al: Reporting and Disclosing Medical Errors: Pediatricians' Attitudes and Behaviors. Arch Pediatr Adolesc Med 2007; 161: 179-85.

15.- Joint Commission International Center for Patient Safety lhttp://www.jcipatientsafety.org/WHO

16.- World Alliance for Patient Safety http://www.who.int/ patientsafety/ worldalliance/en/

17.- Innovation series 2007. IHI Global trigger tool for measuring adverse events http://www.ihi.org/NR/ rdonlyres/B277159C-60D4-4EFD-BF2A-B9FB62CA AA4A/0/ihiglobaltriggertoolwhitepaper 2007.pdf

18.- Institute of healthcare improvement: Improvement Methods. http://www.ihi.org/IHI/Topics/Improvement/ improvementmethods/

19.- American Medical Association Council on Ethical and Judicial Affaire http://www.ama-assn.org/ama/pub/ category/2498.html

20.- National Academy for State Health Policy http://www. nashp.org/Files/Medical_Malpractice_and_Medical Error_Disclosure.pdf

21.- Carta médica de Chile. Sociedad Médica de Santiago. Sociedad Chilena de Medicina Interna. Http://www.carta medica.cl/page.php?5

22.- Proyecto de ley de Derechos y Deberes de las Personas en su Atención de Salud sigue su avance en el Congreso. Http://webhosting.redsalud. Gov.cl/minsal/ noticias/deberespacientes.html

23.- Régimen de Garantías Explícitas en Salud Garantía de Calidad. Estándares mínimos de acreditación para los prestadores institucionales autorizados http://www. minsal.cl/ici/destacados/Standares_A_Cerrada 
definitivos_def_WEB1.pdf

24.- Lester H, Tritter JQ: Medical error: The need to reform medical education. Med Educat 2001; 35: 855-61.

25.- Gallagher TH, Garbutt JM, Waterman AD, et al: Choosing your words carefully. Arch Intern Med 2006; 166: $1585-93$.

26.- Gallagher TH, Studdert D, Levinson W: Disclosing harmful medical errors to patients. N Engl J Med 2007; 356: 2713-9.

27.- Gallagher T, Waterman A, Ebers A, Fraser $V$, Levinson $W$ : Patients' and physicians' attitudes regarding the disclosure of medical errors. JAMA 2003; 281: 1001-7.

28.- Borrell-Carrió F, Páez C, Suñol R, Orrego C, Gil N, Martí $M$ : Errores clínicos y eventos adversos: percepción de médicos de atención primaria. Aten Primaria 2006; 38 (1): 25-32.

29.- Berlinger $N, W u A W$ : Substracting insult from injury: adressing cultural expectations in the disclosure of medical error J Med Ethics 2005; 31: 106-8.

30.- Van Vorst RF, Araya-Guerra R, Felzien M, et al: Rural Community Members' Perceptions of Harm from Medical Mistakes: A High Plains Research Network (HPRN) Study. J Am Board Fam Med 2007; 20: 135-
43.

31.- Proyecto Fondef: "Intervención en crisis" Manual de Primer Apoyo. Pág 16.

32.- Weingart $S$, Pagovich $O$, Sands $D$, et al: What Can Hospitalized Patients Tell Us About Adverse Events? J Gen Intern Med 2005; 20: 830-6.

33.- Miranda F: http://www.medicosparachile.cl/mpch2/ index.php?Option $=$ com_content\&task $=$ view\&id $=77$ \&Itemid $=67$

34.- IOM.Crossing the Quality Chasm: A New Health System for the 21st Century. Washington, DC: National Academies Press; 2001.

35.- National Initiative for Children's Health Care Quality Project Advisory Committee: Principles of patient safety in pediatrics. Pediatrics 2001; 107: 1473-5.

36.- Kraman S, Hamm G: Risk management: extreme honesty may be the best policy. Ann Intern Med 1999; 131: $963-7$

37.- http://163.247.51.38/desarrollo/oirs1/index.php

38.- $W u A W$ : Handling hospital errors: is disclosure the best defense? Annals Intern Med 1999; 131: 970-2.

39.- Suresh G, Horbar JD, Plsek P, et al: Voluntary anonymous reporting of Medical errors for neonatal intensive care. Pediatrics 2004;13: 1609-18. 